\title{
Robust and Reproducible Generation of Induced Neural Stem Cells from Human Somatic Cells by Defined Factors
}

\author{
Tae Hwan Kwak ${ }^{1,2, *}$, Sai Hali ${ }^{1,2, *}$, Sungmin $\mathrm{Kim}^{3}$, Jonghun Kim ${ }^{1}$, Hyeonwoo La ${ }^{4}$, Kee-Pyo Kim ${ }^{5}$, \\ Kwon Ho Hong ${ }^{4}$, Chan Young Shin ${ }^{2}$, Nam-Hyung Kim ${ }^{6}$, Dong Wook Han ${ }^{7}$ \\ ${ }^{1}$ Department of Stem Cell Biology, School of Medicine, Konkuk University, Seoul, Korea \\ ${ }^{2}$ Department of Neuroscience, School of Medicine and Center for Neuroscience Research, Konkuk University, Seoul, Korea \\ ${ }^{3}$ School of Cell and Molecular Medicine, University of Bristol, Bristol, UK \\ ${ }^{4}$ Department of Stem Cell \& Regenerative Biotechnology, Konkuk University, Seoul, Korea \\ ${ }^{5}$ Department of Cell and Developmental Biology, Max Planck Institute for Molecular Biomedicine, Münster, Germany \\ ${ }^{6}$ Department of Animal Sciences, Chungbuk National University, Cheongju, Korea \\ ${ }^{7}$ School of Biotechnology and Healthcare, Wuyi University, Fiangmen, China
}

Background and Objectives: Recent studies have described direct reprogramming of mouse and human somatic cells into induced neural stem cells (iNSCs) using various combinations of transcription factors. Although iNSC technology holds a great potential for clinical applications, the low conversion efficiency and limited reproducibility of iNSC generation hinder its further translation into the clinic, strongly suggesting the necessity of highly reproducible method for human iNSCs (hiNSCs). Thus, in orderto develop a highly efficient and reproducible protocol for hiNSC generation, we revisited the reprogramming potentials of previously reported hiNSC reprogramming cocktails by comparing the reprogramming efficiency of distinct factor combinations including ours.

Methods: We introduced distinct factor combinations, OSKM (OCT4+SOX2+KLF4+C-MYC), OCT4 alone, SOX2 alone, SOX2+HMGA2, BRN4+SKM+SV4OLT $\left(\mathrm{BSKM}^{\mathrm{LT}}\right), \mathrm{SK}^{\mathrm{LT}}, \mathrm{SM}^{\mathrm{LT}}$, and $\mathrm{SKM}^{\mathrm{LT}}$ and performed comparative analysis of reprogramming potentials of distinct factor combinations in hiNSC generation.

Results: Here we show that ectopic expression of five reprogramming factors, BSKM ${ }^{\mathrm{LT}}$ leads the robust hiNSC generation (>80 folds enhanced efficiency) from human somatic cells compared with previously described factor combinations. With our combination, we were able to observe hiNSC conversion within 7 days of transduction. Throughout further optimization steps, we found that both BRN4 and KLF4 are not essential for hiNSC conversion. Conclusions: Our factor combination could robustly and reproducibly generate hiNSCs from human somatic cells with distinct origins. Therefore, our novel reprogramming strategy might serve as a useful tool for hiNSC-based clinical application.

Keywords: Direct conversion, Human induced neural stem cells, Robust and reproducible generation, Defined factors

\footnotetext{
Received: August 7, 2019, Revised: August 16, 2019, Accepted: August 19, 2019, Published online: February 29, 2020

Correspondence to Dong Wook Han

School of Biotechnology and Healthcare, Wuyi University, Pengjiang Disctrict, Jiangmen 529020, China

Tel: +82-43-261-2546, Fax: +82-43-261-3723

E-mail: dwhan.stem@gmail.com

*These authors contributed equally to this work.

(c) This is an open-access article distributed under the terms of the Creative Commons Attribution Non-Commercial License (http://creativecommons.org/ licenses/by-nc/4.0/), which permits unrestricted non-commercial use, distribution, and reproduction in any medium, provided the original work is properly cited.

Copyright (c) 2020 by the Korean Society for Stem Cell Research
} 


\section{Introduction}

Neural stem cells (NSCs), one of somatic stem cell types, can differentiate into various types of neurons and glial cells of the central nervous system (CNS) in response to distinct signaling cues (1) and their transplantation could reverse the pathophysiology of CNS disorders in animal models (2). Thus, NSCs have long been considered as a highly suitable cell source for restoring damaged nerve tissues in various neurodegenerative diseases $(3,4)$. Despite the clinical potentials of the brain tissue-derived NSCs, their limited accessibility and the potential risk of immune rejection upon allogeneic transplantation may preclude their clinical application. Recent induced pluripotent stem cell (iPSC) technology (5) open a new avenue of patient-specific clinical applications such as cell replacement therapy. Since iPSCs are equivalent to embryonic stem cells (ESCs) in their self-renewal capacity as well as their pluripotency, they have been considered as an alternative cell source for transplantable patient-specific NSCs. However, there are also some roadblocks of iPSC-based cell therapy such as the potential risk of tumor formation upon transplantation due to the residual undifferentiated pluripotent stem cells even after long differentiation steps.

Alternatively, ectopic expression of cell type-specific genes could lead a direct cell fate transition from a somatic cell state into completely distinct cellular identities without first generating iPSCs (6-12). This direct conversion technology could also be a useful tool to generate patient-specific NSCs which are non-tumorigenic (10, 13, 14). Some previous studies have used the forced expression of the Yamanaka's reprogramming factors, OCT4, SOX2, KLF4, and C-MYC (OSKM), to convert somatic cells into human induced neural stem cells (hiNSCs) with a combined treatment of small molecules which can facilitate the reprogramming procedure $(15,16)$. However, the iNSC conversion process using OSKM could involve a transiently acquired pluripotent state, suggesting the potential risk of tumor formation using this reprogramming strategy (17). In other cases of hiNSC generation, reprogramming could be achieved by overexpression of a single transcription factor (18-20) or a combination of cell type-specific genes (21). Those directly reprogrammed hiNSCs are non-tumorigenic upon transplantation to the animal model (18-20). Nevertheless, the extremely low efficiency as well as unclear reproducibility of iNSC generation have been remained as a major roadblock of hiNSC technology $(15,19,21,22)$.
Previously we have also described the generation of iNSCs from mouse fibroblasts through the ectopic expression of NSC-specific transcription factors, Brn4, Sox2, Klf4, and c-Myc (BSKM) $(10,11)$. The directly converted iNSCs are nearly indistinguishable from the brain tissue-derived NSCs in their morphology, gene expression patterns, epigenetic status, self-renewal capacity, and both in vitro and in vivo multipotency $(10,11,14,23)$. Moreover, iNSC could ameliorate the disease phenotypes upon transplantation into animal models without forming tumor, showing their therapeutic potentials for CNS diseases $(14,24)$. More importantly, we recently demonstrated that the BSKM-mediated iNSC generation is a direct process that does not involve an intermediate pluripotent state (25), indicating that BSKM may be a highly reliable and safe reprogramming factor combination for generating transplantable hiNSCs. However, the reprogramming capacity of BSKM in human cells has yet to be determined (26).

In the current study, we have established an efficient and reproducible method for generating hiNSCs using fibroblasts from multiple origins by introducing a defined and optimized set of reprogramming factors, resulting in a robust hiNSC conversion within 7 days of transduction. The transdifferentiated hiNSCs exhibited typical features of NSCs such as morphology, gene expression patterns, and differentiation capacity. Our robust and reproducible reprogramming strategy for hiNSC generation may facilitates hiNSC-based clinical translation.

\section{Materials and Methods}

\section{Cell culture}

Human fibroblasts maintained in DMEM with high glucose (Welgene) containing 10\% fetal bovine serum (Seradigm), $1 \times$ MEM/NEAA (Gibco), and $1 \times$ penicillin/ streptomycin/glutamine (Gibco). The ESC-derived NSCs were maintained in DMEM/F12 (Corning) supplemented with $100 \times \mathrm{N} 2$ supplement (Gibco), 50× B27 without vitamin A (Gibco), $1 \%$ penicillin/streptomycin (PS) (Gibco), $1 \%$ GlutaMAX (Gibco), $55 \mu \mathrm{M} \beta$-mercaptoethanol (Gibco), $0.05 \%$ Bovine serum albumin fraction $\mathrm{V}$ (Invitrogen), 10 $\mathrm{ng} / \mathrm{ml}$ leukemia inhibitory factor, $3 \mu \mathrm{M}$ CHIR99021 (Tocris), and $2 \mu \mathrm{M} \mathrm{SB} 431542$ (Sigma). hiNSCs were generated and maintained in neural stem cell expansion medium (NSCEM), composed of ReNcell NSC Maintenance Medium (Merck) supplemented with $20 \mathrm{ng} / \mathrm{ml}$ bFGF (Peprotech), $20 \mathrm{ng} / \mathrm{ml}$ EGF (Peprotech), $0.5 \mu \mathrm{M}$ phorbol-12-myristate-13-acetate (Sigma), $3 \mu$ M CHIR99021 
(Tocris), and $2 \mu \mathrm{M} \mathrm{SB} 431542$ (Sigma). The H9 hESCs were maintained in $\mathrm{TeSR}^{\mathrm{TM}}-\mathrm{E}^{\mathrm{TM}}$ medium (STEMCELL Technologies) and subcultured using the ReLeSR ${ }^{\mathrm{TM}}$ (STEMCELL Technologies) according to the manufacturer's instructions. Differentiation of hESC into NSCs was performed as previously described (27). Briefly, hESCs were detached by ReleSR ${ }^{\mathrm{TM}}$ (STEMCELL Technologies) to form the Embryoid bodies (EBs) and EBs were cultured in the neural induction medium (NIM) containing DMEM/F12 (Corning) supplemented with 1x N2 (Gibco), 1× B27 without vitamin A (Gibco), 1\% penicillin/streptomycin (Gibco), 1\% GlutaMAX ${ }^{\mathrm{TM}}$ (Gibco), $1 \%$ NEAA (Gibco), $55 \mu \mathrm{M} \beta$-mercaptoethanol (Gibco) in the presence of $3 \mu \mathrm{M}$ CHIR99021, $2 \mu \mathrm{M} \mathrm{SB} 431542$ and $10 \mathrm{ng} / \mathrm{ml}$ hLIF (Millipore). After 7 days, the EBs were dissociated into single cells using $\operatorname{TrypLE}^{\mathrm{TM}}$ (Gibco) and re-plated on the Matrigel (Corning)-coated surfaces. The hESC-derived NSCs were stabilized in NIM by serial passaging.

\section{Retrovirus production}

Retroviral particles were produced by transfection of pMX vectors encoding reprogramming factors $(10 \mu \mathrm{g})$ into Platinum E cells (Cell Biolabs) using $30 \mu 1$ of jetPEI transfection reagent (Polyplus). After $48 \mathrm{hrs,} \mathrm{the} \mathrm{super-}$ natants containing viral particles were carefully collected and filtered through a $0.22-\mu \mathrm{m}$ syringe filter (Minisart).

\section{Generation of hiNSCs}

To generate hiNSCs, $5 \times 10^{4}$ human fibroblasts were plated onto a gelatin-coated $35 \mathrm{~mm}$ dish. Next day, fibroblasts were transduced with retroviral particles encoding a reprogramming factor combination using $6 \mu \mathrm{g} / \mathrm{ml}$ protamine sulfate (Sigma), re-plated onto a Matrigel (Corning)coated $35 \mathrm{~mm}$ dish and cultured in NSCEM, which was replaced with fresh medium every other day. Once the initial colonies were observed, stable hiNSC lines were established by serial passaging. For the comparative analysis of reprogramming potentials of distinct factor combinations in hiNSC generation, we used the identical conditions from previous studies for each reprogramming cocktails, OSKM (15), OCT4 (19), SOX2 (18), and SOX2+HMGA2 (21).

\section{Gene expression analysis}

Total RNA was isolated by using a Hybrid-RTM RNA isolation kit (GeneAll) according to the manufacturer's protocol. RNA (1 $\mu \mathrm{g})$ was converted to cDNA using a High Capacity cDNA Reverse Transcription Kit (Applied Biosystems). RT-PCR was performed using GoTag Green
Master Mix (Promega). qPCR was performed using SYBR Green PCR Master Mix (Applied Biosystems) on a QuantStudio 3 Real-Time PCR instrument (Applied Biosystems). $\Delta \mathrm{Ct}$ values were calculated by subtracting the $G A P D H \mathrm{Ct}$ value from that of each target gene. Relative expression levels were calculated by using the $2^{-\Delta \Delta \mathrm{Ct}}$ method. The primer sets are listed in Supplementary Table S1.

\section{RNA sequencing analysis}

Quality of the RNA-seq raw reads were assessed with Fastqc (0.11.8) and the reads were aligned with STAR (v2.6.1a) on UCSC hg38 human genome. Gene expression level was calculated and normalized with Cuffnorm (v2.2.1, Cufflinks). Genes with more than two-fold difference of expression levels between hFFs and hESC-derived NSCs and FPKM value of more than 2 were chosen to generate the heatmap. Selected genes were sorted to be clustered into different groups of up-regulated or downregulated genes compared to hESC-derived NSCs. Data visualization was conducted with heatmap. 2 function of $\mathrm{R}$ package gplots (v3.0.1.1).

\section{Immunocytochemistry}

Cells were fixed with $4 \%$ paraformaldehyde (Chemcruz) for $15 \mathrm{~min}$ at room temperature, and then washed three times with PBS (Life Genomics). After washing, the fixed cells were permeabilized and blocked with PBS containing $0.03 \%$ Triton X-100 (Sigma) and 6\% BSA (Sigma) for 1 $\mathrm{hr}$ at room temperature. The following primary antibodies were used: goat anti-SOX2 (Santa Cruz, $1: 200$ ), goat anti BRN2 (Santa Cruz, $1: 200$ ), rabbit anti-BLBP (Santa Cruz, $1: 200$ ), rat anti-MSI1 (MBL, $1: 200$ ), rabbit anti-MSI2 (Abcam, $1: 200$ ), mouse anti-TUY1 (Covance, 1 : 500), rabbit anti-GFAP (Dako, $1: 500$ ), mouse anti-MBP (Abcam, $1: 500$ ), rabbit anti-GABA (Sigma, $1: 200$ ), rabbit anti-GLU (Sigma, $1: 200$ ), goat-anti ChAT (Merck, $1: 200$ ), and rabbit anti-TH (Merck, $1: 200)$, and rabbit anti-MBP (Abcam, $1: 200$ ). Permeabilized cells were incubated with primary antibodies for $16 \mathrm{hrs}$ at $4^{\circ} \mathrm{C}$, washed three times with PBS, and incubated with secondary antibodies for $2 \mathrm{hrs}$ at room temperature. Counterstaining was performed with Hoechst 33342 (Sigma).

\section{In vitro differentiation of NSCs}

To differentiate hiNSCs and hESC-derived NSCs into neurons, the cells were dissociated into single cells and $1 \times 10^{4}$ cells were plated onto Matrigel-coated single well of 4-well plate. After $24 \mathrm{hrs,} \mathrm{the} \mathrm{medium} \mathrm{was} \mathrm{replaced}$ with a $1: 1 \mathrm{mix}$ of DMEM/F12 (Corning) and Neurobasal 
Medium (Gibco) supplemented with $100 \times$ N2 supplement (Gibco), 50× B27 (Gibco), 1\% PS (Gibco), 1\% GlutaMAX (Gibco), 1\% NEAA (Gibco), $55 \mu \mathrm{M} \beta$-mercaptoethanol (Gibco), $1 \mu \mathrm{g} / \mathrm{ml}$ heparin (Sigma), $10 \mathrm{ng} / \mathrm{ml} \mathrm{BDNF}$ (Peprotech), 10 ng/ml GDNF (Peprotech), $200 \mu \mathrm{M}$ ascorbic acid (Peprotech), and $125 \mu \mathrm{M}$ cAMP (Peprotech). To differentiate hiNSCs and hESC-derived NSCs into astrocytes, the cells were dissociated and plated as above. After 24 hrs, the medium was replaced with DMEM/F12 (Corning) supplemented with $100 \times \mathrm{N} 2$ supplement (Gibco), 50× B27 (Gibco), 1\% PS (Gibco), 1\% GlutaMAX (Gibco), 1\% NEAA (Gibco), 10 ng/ml BMP4 (Peprotech), and $20 \mathrm{ng} / \mathrm{ml} \mathrm{CNTF} \mathrm{(Peprotech).} \mathrm{Two} \mathrm{weeks} \mathrm{after} \mathrm{induc-}$ ing differentiation, the neurons and astrocytes were immunostained with antibody against TUJ1 and GFAP, respectively. The subtypes of neurons were determined at 5 weeks after inducing differentiation.

\section{Statistical analysis}

All information related to statistical tests is documented in the corresponding figure legends and in the supplementary figure legends. Data are reported as mean values from at least three replicates, with error bars denoting SD. Statistical significance was evaluated with unpaired twotailed Student's t-test.

\section{Results}

\section{Robust and reproducible generation of hiNSCs}

During the early phase of reprogramming, an enhanced proliferation rate of somatic cells has been shown to be critical for achieving improved reprogramming efficiency $(28,29)$. This prompted us to hypothesize that the addition of NSC-specific mitogens which can activate cell cycle may elevate the direct conversion efficiency toward NSCs. To this end, the human fetal fibroblasts (hFFs) transduced with BSKM $(10,11)$ were cultured in neural stem cell expansion medium (NSCEM) supplemented with EGF and bFGF and three additional small molecules; phorbol-12-myristate-13-acetate, CHIR99021, and SB431542, which are all known to activate a mitotic pathway in early-stage progenitors of the neuroectoderm (30, 31) (Fig. 1A). However, BSKM-transduced hFFs did not show any morphological changes until 4 weeks after viral infection. Most of the transduced hFFs maintained their typical fibroblast morphology with a slow proliferation rate and eventually underwent cellular senescence (data not shown). This finding suggests that BSKM is not sufficient to convert human fibroblasts into an NSC-like state.

Previous studies declaimed that the forced expression of
SV40 large T antigen (SV4OLT) significantly enhances the reprogramming efficiency of human iPSCs by improving cell proliferation rates and also by reducing cellular apoptosis (32). Additional SV40LT could also facilitate the transdifferentiation and expansion of human induced hepatocytes (12). Thus, to improve the conversion efficiency of hiNSCs, we introduced the SV4OLT into hFFs together with BSKM (Fig. 1A). After 7 days of viral infection, we found the initial iNSC clusters with typical epithelial morphology and higher proliferation capacity compared with those of unreprogrammed hFFs $(12.25 \pm 9.56$ colonies per individual experimental group) (Fig. 1A). At 2 weeks of transduction, a NSC-like cell population with homogenous morphology were observed and they could be stably expanded along the serial passaging (Fig. 1B and 1C). A subset of NSC markers started to be expressed in established hiNSCs (passage 1) with complete suppression of $\mathrm{hFF}$ markers and became strongly activated upon further passaging (passage 10) (Fig. 1D), indicating the gradual reprogramming process of hiNSCs as in mouse case (10, 11). Similarly, hiNSCs generated with BSKM and SV40LT exhibited the gradual morphological changes during further passaging, i.e., less defined shapes in early passage and well-defined and typical NSC-like shapes at later passages (Fig. 1C) (hereafter referred to BSKM ${ }^{\mathrm{LT}}$ hiNSCs).

Although previous studies demonstrated the direct conversion of human somatic cells into hiNSCs, the reproducibility of previous reprogramming cocktails for generating hiNSCs using human somatic cells from distinct origins has yet to be determined $(18,20)$, strongly suggesting the necessity of highly reproducible method for hiNSCs. Thus, we first tried to evaluate the reproducibility of our reprogramming factor combination (BSKM plus SV4OLT) in hiNSC generation. For this, we introduced our factor combination into two lines of human fibroblasts, CRL2097 human dermal fibroblasts (obtained from American Type Culture Collection, Manassas, VA, USA) and F134 human adult fibroblasts (33), which are derived from distinct individuals. Similar with hFFs (Fig. 1C and 1D), both CRL-2097 and F134 fibroblasts could be reprogrammed into hiNSCs (Supplementary Fig. S1A and S1C) expressing multiple NSC markers (Supplementary Fig. S1B and S1D). Taken together, our data indicate that our reprogramming cocktail is highly robust and reproducible for generating hiNSCs from distinct genetic backgrounds.

BSKM $^{\mathrm{LT}}$ hiNSCs displayed the specific morphological characteristics of radial glial cells in the ventricular zone of the developing brain (Fig. 1C). To further assess the regional identity of BSKM ${ }^{\mathrm{LT}}$ hiNSCs, we next checked the 
A

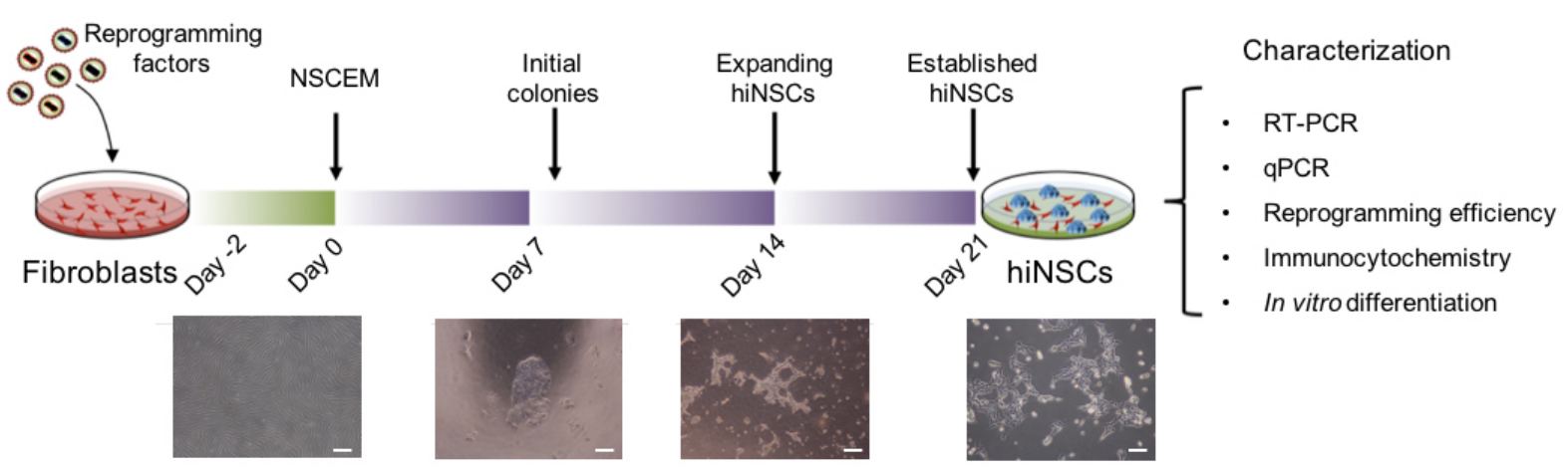

B
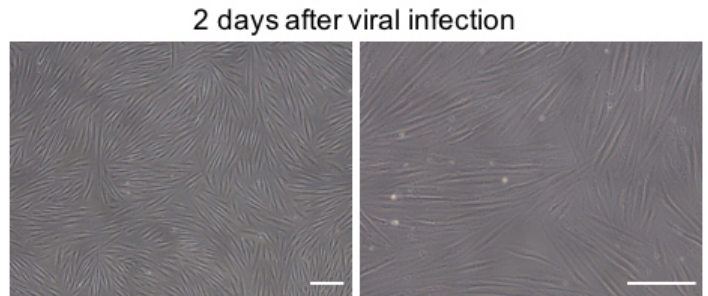

C
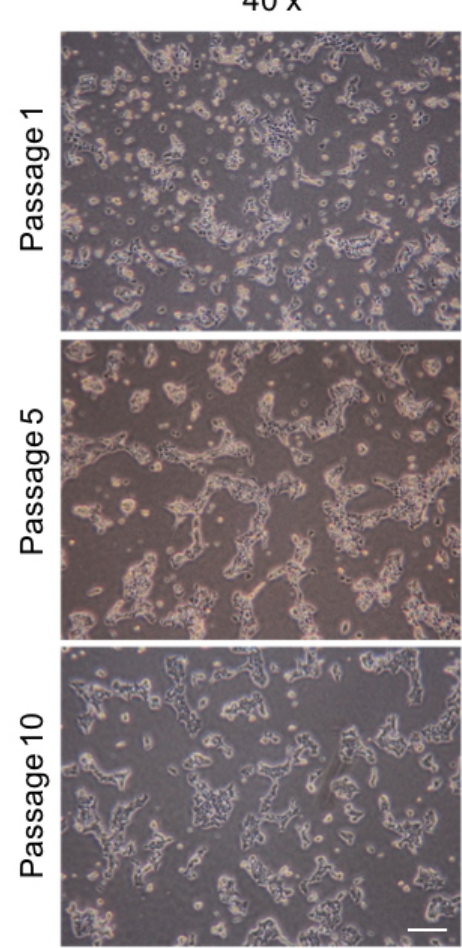

$100 x$
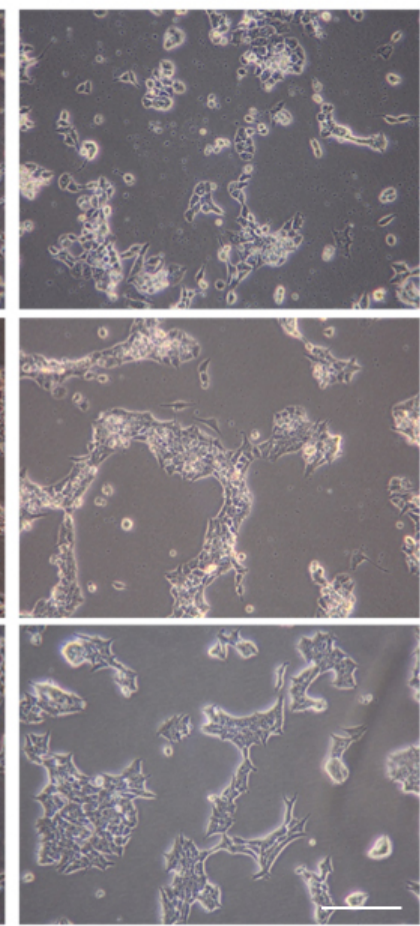

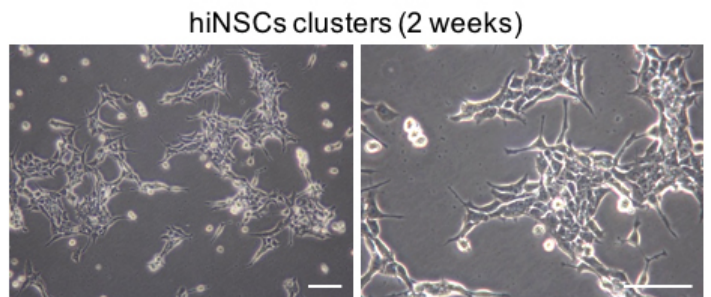

D

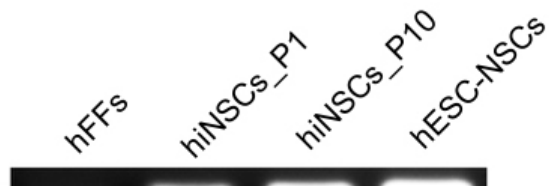

SOX2

PAX6

HEY2

BRN2

ASCL1

MSI1

MSI2

MAP2

$B L B P$

GLAST

PROM1

PLZF

RC1

CXCR 4

PDGFRB

THY1

GAPDH

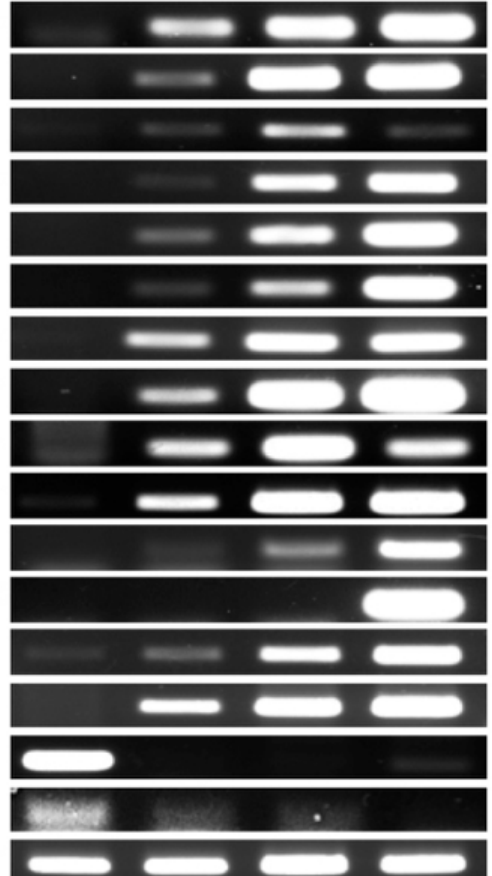

Fig. 1. Generation of hiNSCs from hFFs using BSKM with SV4OLT. (A) Schematic illustration of the reprogramming procedure for generating hiNSCs. Morphological changes during the reprogramming period are shown. Scale bars, $100 \mu \mathrm{m}$. (B) Morphology of hiNSCs after 2 weeks of transduction. Scale bars, $100 \mu \mathrm{m}$. (C) Morphology of the established hiNSCs at different passages. Scale bars, $100 \mu \mathrm{m}$. (D) Expression pattern of NSC- and fibroblast-specific markers were analyzed by RT-PCR in early and later passages of hiNSCs. GAPDH was used as a positive control. 
expression patterns of region-specific maker genes which play critical roles in establishing the precise domain boundaries of the developing nervous system (34). Notably, the expression pattern of certain genes such as PAX6, BRN2, MSI1, PROMI, and RC1 varied among the BSKM ${ }^{\mathrm{LT}}$ hiNSC lines, although the pan-NSC markers such as SOX2, HEY2, MSI2, BLBP, CXCR4, and GLAST were commonly expressed (Fig. 1D, Supplementary Fig. S1B and S1D). These data could be explained by the heterogeneity of reprogrammed BSKM ${ }^{\mathrm{LT}}$ hiNSCs, which represent distinct regional identities and developmental stages of NSCs. Despite the heterogeneous identity of BSKM ${ }^{\mathrm{LT}}$ hiNSCs, all BSKM ${ }^{\mathrm{LT}}$ hiNSC lines from distinct origins were negative for $P L Z F$, a transcription factor that functions in early neurodevelopment and disappears in the late stage of neurodevelopment (35), supporting that BSKM ${ }^{\mathrm{LT}}$ hiNSCs more likely represent late-stage radial glial cells in developing brain (Fig. 1D, Supplementary Fig. S1B and S1D).

\section{A}

\begin{tabular}{|c|c|c|c|c|c|c|c|c|c|}
\hline Control & Lu et al., 2013 & Zhu et al., 2013 & $\begin{array}{c}\text { Ring et al., } \\
2012\end{array}$ & Yu et al., 2015 & \multicolumn{5}{|c|}{ Current Protocols } \\
\hline \multirow[t]{2}{*}{ MOCK } & \multirow{2}{*}{$\begin{array}{c}\text { OCT4 } \\
+ \text { SOX2 } \\
+ \text { KLF4 } \\
+ \text { C-MYC }\end{array}$} & \multirow[t]{2}{*}{ OCT4 } & \multirow[t]{2}{*}{ sox2 } & \multirow[t]{2}{*}{$\begin{aligned} & \text { SOX2 } \\
+ & \text { HMGA2 }\end{aligned}$} & Mock & $\begin{array}{l}\text { SOX2 } \\
+ \text { KLF4 }\end{array}$ & $\begin{aligned} & \text { SOX2 } \\
+ & \text { C-MYC }\end{aligned}$ & $\begin{array}{c}\text { SOX2 } \\
+ \text { KLF4 } \\
+ \text { C-MYC }\end{array}$ & $\begin{array}{c}\text { BRN4 } \\
+ \text { SOX2 } \\
+ \text { KLF4 } \\
+ \text { C-MYC }\end{array}$ \\
\hline & & & & & \multicolumn{5}{|c|}{ +SV40LT } \\
\hline
\end{tabular}
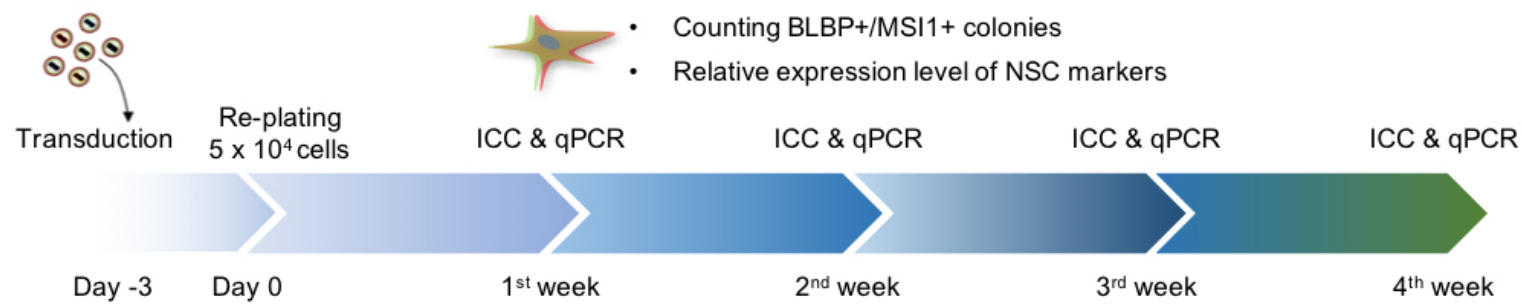

$\begin{array}{ccc}\text { Day }-3 \quad \text { Day } 0 & 1^{\text {st }} \text { week } & 2^{\text {nd }} \text { week } \\ & \begin{array}{c}\text { Inducing reprogramming } \\ \text { (NSCEM) }\end{array} & \text { Cell line establishment }\end{array}$

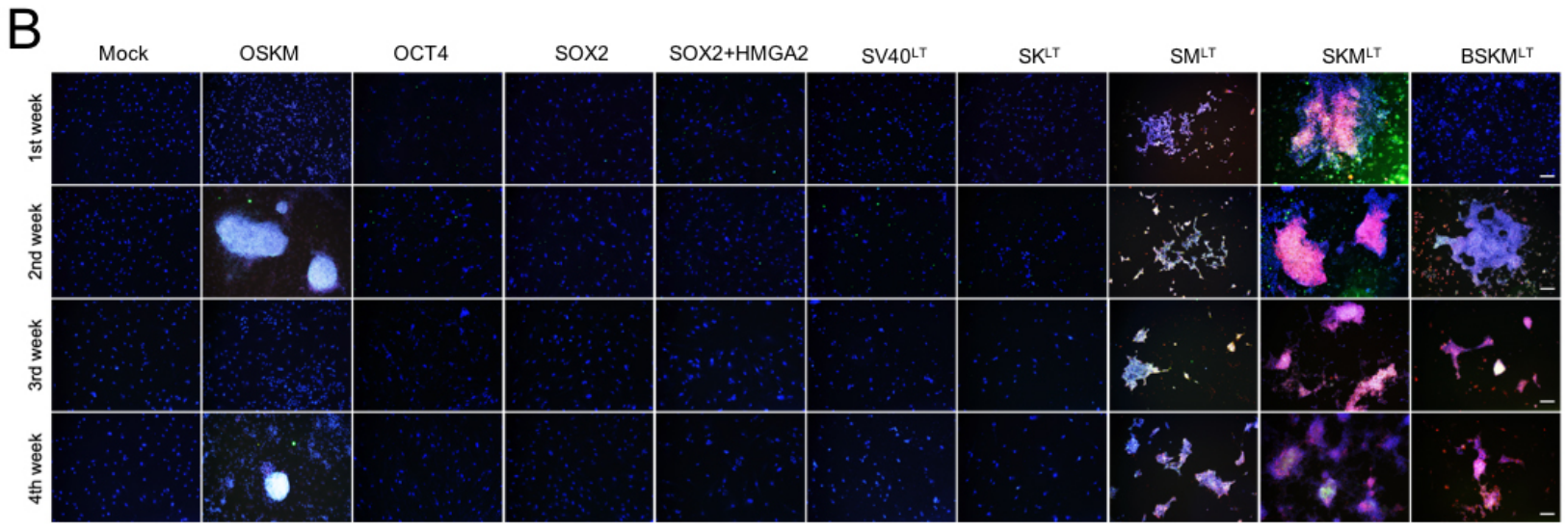

Fig. 2. Comparative analysis of reprogramming potentials of distinct factor combinations. (A) The schematic illustration depicting the strategy for comparing the reprogramming efficiency of distinct factor combinations. (B) Time-course immunofluorescence analysis for comparing reprogramming potentials of distinct factor combinations. Scale bars, $100 \mu \mathrm{m}$. (C) Morphology of hiNSC clusters at 2 weeks after transduction. Scale bars, $100 \mu \mathrm{m}$. (D) The number of $B L B P^{+} / M S I 1^{+}$colonies were counted in a time-course manner. Data are presented as mean \pm SD from six independent experiments. ${ }^{*} \mathrm{p}<0.05, * \mathrm{p}<0.01,{ }^{*} * \mathrm{p}<0.001$. 


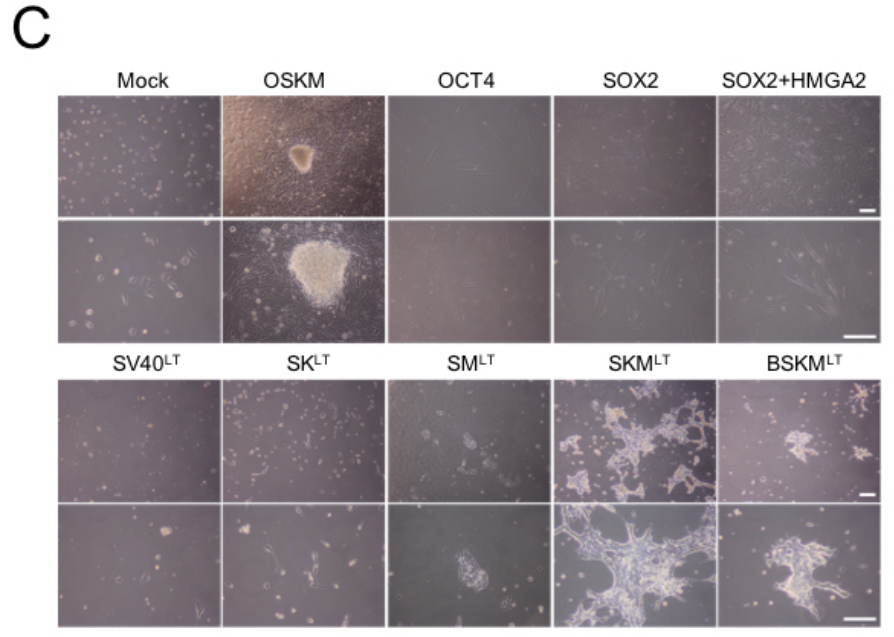

Fig. 2. Continued.

\section{Comparative analysis of reprogramming potentials of distinct factor combinations in hiNSC generation}

Previous studies claimed that the reprogramming efficiency toward hiNSCs was less than $0.96 \%$ and the time required for hiNSC generation was around 20 days (up to 35 days) (Supplementary Table S2) (15, 18, 19, 21, 22). Thus, to develop a highly efficient and reproducible protocol for hiNSC generation, we revisited the reprogramming potentials of previously reported hiNSC reprogramming cocktails by comparing the reprogramming efficiency of distinct factor combinations including ours. For this, we introduced distinct factor combinations, OSKM $(15,16$, 22), OCT4 alone (19, 20), SOX2 alone (18, 20), SOX2+ HMGA2 (21), and BSKM plus SV4OLT. In addition, to scale down the number of factors required for hiNSC generation, we also introduced the following combinations, SOX2 + KLF4 +SV4OLT $\left(\mathrm{SK}^{\mathrm{LT}}\right), S O X 2+C-M Y C+S V 4 O L T$ $\left(\mathrm{SM}^{\mathrm{LT}}\right)$, and SOX2+KLF4+C-MYC+SV4OLT $\left(\mathrm{SKM}^{\mathrm{LT}}\right)$ (Fig. 2A).

On day 2 post-infection of each combination, $5 \times 10^{4}$ transduced cells were re-plated onto Matrigel-coated plates and cultured in NSCEM (Fig. 2A). For a fair comparison, the expression levels of each exogenous factors were determined by qPCR on day 5 of transduction. The qPCR analysis showed that each exogenous reprogramming factor was successfully introduced and stably expressed in the transduced hFFs (Supplementary Fig. S2A). To perform an unbiased comparative analysis of direct conversion efficiency using distinct combinations in a time-course manner, we immunoassayed iNSC colonies using antibodies against NSC-specific proteins (BLBP and MSII) and the

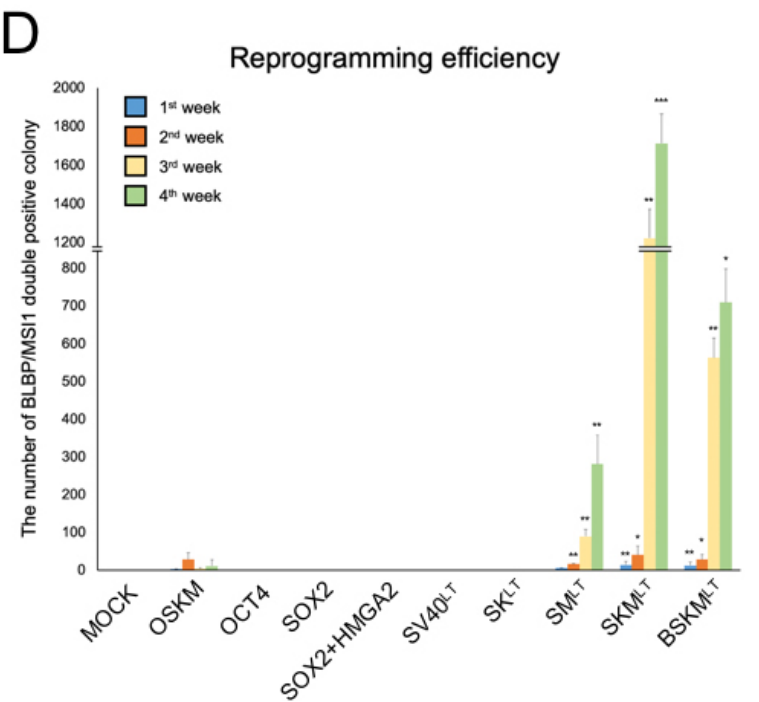

numbers of $\mathrm{BLBP}^{+} / \mathrm{MSII}^{+}$colonies were counted every week for 4 weeks of reprogramming period (Fig. 2A). OSKM, SM ${ }^{\mathrm{LT}}, \mathrm{SKM}^{\mathrm{LT}}$, and $\mathrm{BSKM}^{\mathrm{LT}}$ groups but not others exhibited $\mathrm{BLBP}^{+} / \mathrm{MSII}^{+}$colonies with substantial morphological changes after 2 weeks post infection (Fig. 2B, $2 \mathrm{C}$ and Supplementary Fig. S2B). Although we found some $B L B P^{+} / \mathrm{MSII}^{+}$colonies in OSKM-transduced $\mathrm{hFFs}$, the number of $B L B P^{+} / M S I 1^{+}$colonies was significantly decreased and nearly no colony remained after 4 weeks (Fig. 2B and 2D), suggesting that OSKM may be insufficient to stably maintain a NSC-like state in the reprogrammed cells in our experimental setting. In contrast, the number of both $B L B P$ and $M S I$ expressing colonies was dramatically increased in hFFs transduced with $\mathrm{SM}^{\mathrm{LT}}, \mathrm{SKM}^{\mathrm{LT}}$, and $\mathrm{BSKM}^{\mathrm{LT}}$ upon further passaging (Fig. $2 \mathrm{~B}$ and 2D). Surprisingly, we found $B L B P^{+} / \mathrm{MSI}^{+}$colonies from $\mathrm{SKM}^{\mathrm{LT}}$ transduced hFFS within the first week of transduction (Fig. 2B) and the colony numbers were exponentially increased in the third and fourth weeks of reprogramming (Fig. 2D). This data indicates that BRN4 withdrawal rather facilitates the direct conversion process toward hiNSCs. Notably, $\mathrm{SM}^{\mathrm{LT}}$ condition in which both $B R N 4$ and KLF4 were omitted also exhibited the rapid conversion into hiNSCs within 7 days of transduction (Fig. 2B), although the colony number was slightly lower compared with those of SKM ${ }^{\mathrm{LT}}$ and BSKM ${ }^{\mathrm{LT}}$ (Fig. 2D). Taken together, our time-course analysis indicates that both BRN4 and KLF4 are not essential for hiNSC generation.

Our time-course immunostaining analysis detects fully reprogrammed hiNSCs expressing both BLBP and MSII. 


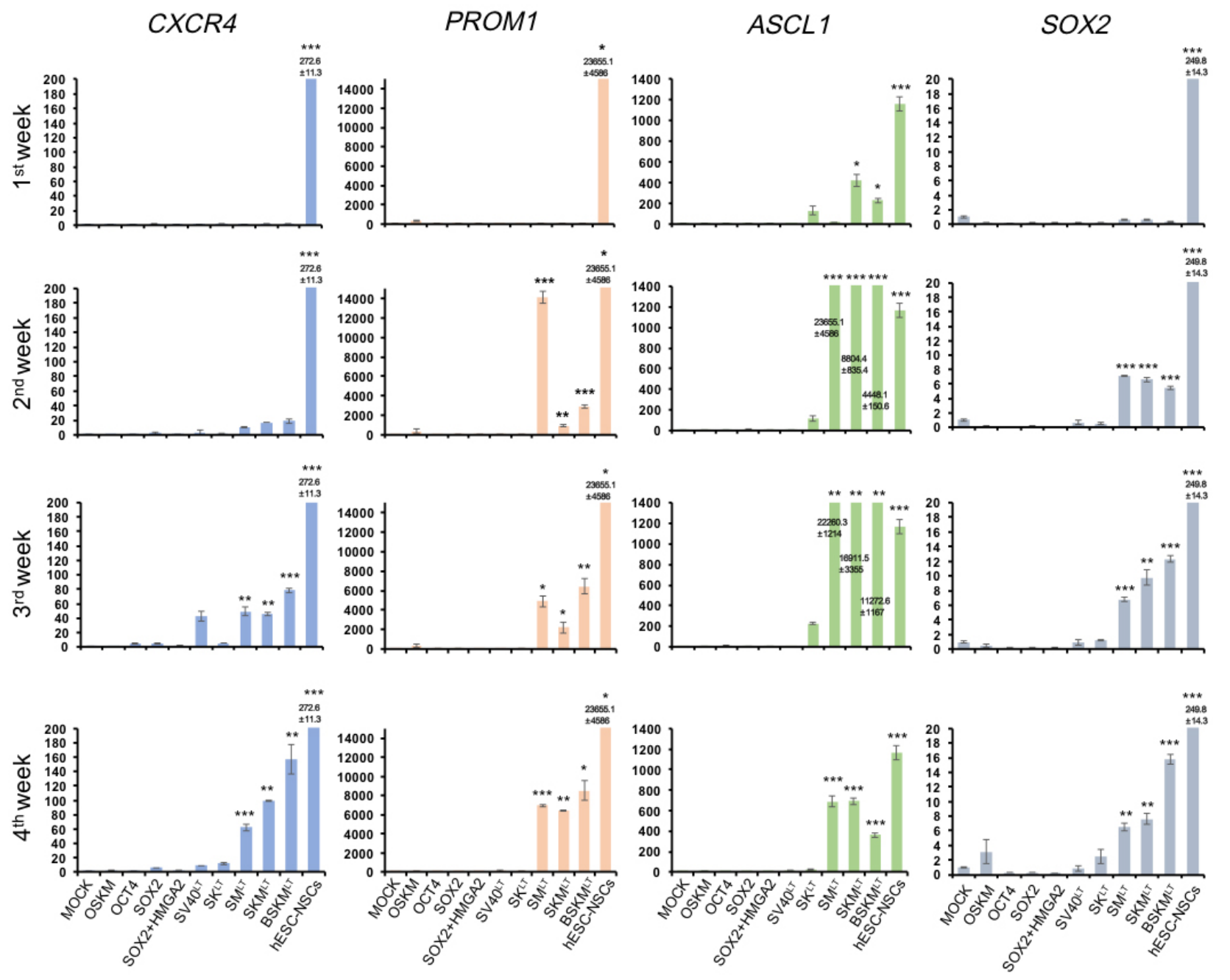

Fig. 3. Expression pattern of NSC-specific markers during hiNSC generation. Expression pattern of NSC markers in hFFs transduced with distinct factor combinations was analyzed by qPCR in a time-course manner. All the values were normalized to those of non-transduced hFFs. Data are presented as mean \pm SD of triplicate values. ${ }^{*} \mathrm{p}<0.05, * * \mathrm{p}<0.01,{ }^{* * *} \mathrm{p}<0.001$.

Thus, it is likely that we may miss intermediate reprogrammed cells which express $B L B P$ and MSI1 partially or only on mRNA level upon introduction of some reprogramming cocktails which failed to generate $B L B P^{+}$/ $\mathrm{MSI1}^{+}$colony. To exclude this possibility, we next performed qPCR analysis to explore the early molecular changes upon the introduction of reprogramming incompetent (OKSM, OCT4 alone, SOX2 alone, SOX2+ $H M G A 2$, and SV4OLT alone) and competent $\left(\mathrm{SM}^{\mathrm{LT}}\right.$, $\mathrm{SKM}^{\mathrm{LT}}$, and $\mathrm{BSKM}{ }^{\mathrm{LT}}$ ) factor combinations. For this, we introduced distinct factor combinations in hFFs and determined the expression levels of endogenous NSC markers including CXCR4, PROM1, ASCL1, and SOX2 every week (up to 4 weeks). Consistent with our immunostaining data, NSC markers were strongly activated in $\mathrm{SM}^{\mathrm{LT}}$, $\mathrm{SKM}^{\mathrm{LT}}$, and BSKM ${ }^{\mathrm{LT}}$ transduced hFFs (Fig. 3). On the other hand, other reprogramming incompetent cocktails (OSKM, OCT4 alone, SOX2 alone, SOX2+HMGA2, and SV40LT alone) failed to activate NSC markers even after 4 weeks of transduction (Fig. 3), supporting our immunostaining data (Fig. 2B and 2D). Collectively, our data indicate that our factor combinations $\left(\mathrm{SM}^{\mathrm{LT}}, \mathrm{SKM}^{\mathrm{LT}}\right.$, and $\mathrm{BSKM}^{\mathrm{LT}}$ ) led robust and reproducible hiNSC generation, and $\mathrm{SKM}^{\mathrm{LT}}$ combination, the optimized factor combination in this study, exhibited the highest conversion efficiency compared with those of $\mathrm{SM}^{\mathrm{LT}}$ and $\mathrm{BSKM}^{\mathrm{LT}}$.

\section{hiNSCs share molecular and functional features with hESC-derived control NSCs}

To characterize $\mathrm{SKM}^{\mathrm{LT}}$ hiNSCs, we generated five hiNSC lines from three independently transduced hFFs with $\mathrm{SKM}^{\mathrm{LT}}$. All the hiNSC lines displayed typical mor- 

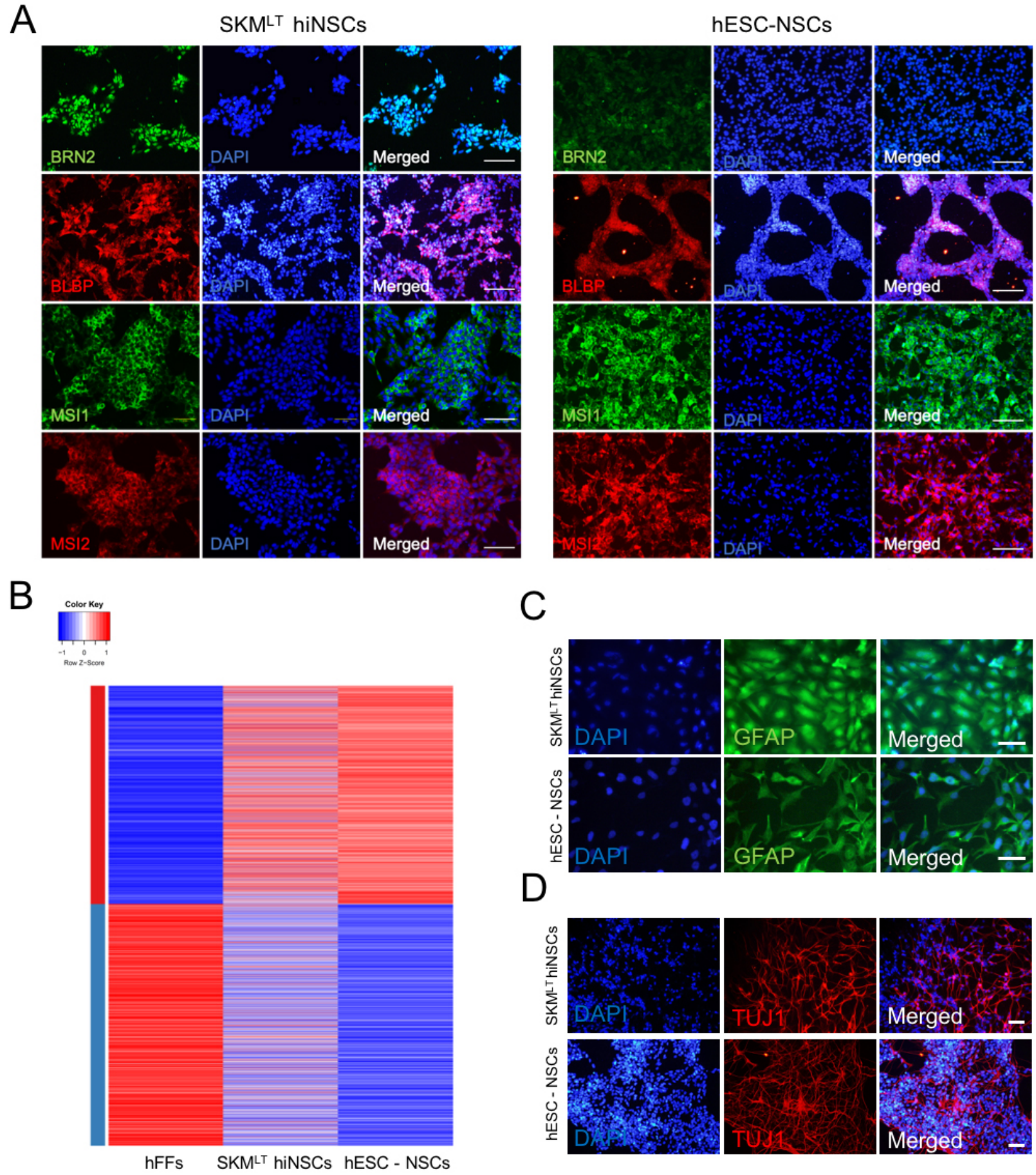

D

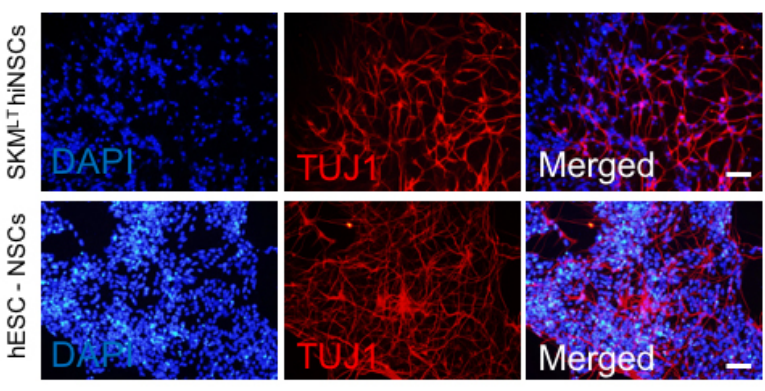

Fig. 4. Characterization of SKM ${ }^{\mathrm{LT}}$ hiNSCs. (A) Immunofluorescence images of hESC-derived NSCs and $\mathrm{SKM}^{\mathrm{LT}}$ hiNSCs using antibodies against $B R N 2, B L B P, M S I 1$, and MSI2. Scale bars, $100 \mu \mathrm{m}$. (B) A heat map representing expression profile of genes with more than two-fold

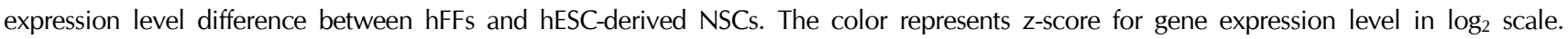
Clusters with red and blue bar on the left represent genes with lower and higher expression in hFFs compared to hESC-derived NSCs, respectively. (C, D) Differentiation potential of SKM ${ }^{\mathrm{LT}}$ hiNSCs into astrocytes (C) and neurons (D) as determined by immunocytochemistry with antibodies against GFAP and TUJ1, respectively, Scale bars, $100 \mu \mathrm{m}$. (E, F) The efficiency of differentiation into astrocytes (E) and neurons (F) from hESC-derived NSCS and SKM ${ }^{\mathrm{LT}}$ hiNSCS was quantified and compared via immunostaining with GFAP and TUJ1, respectively. Data are presented as mean \pm SD from eight independent experiments. N.S.: not significant. 


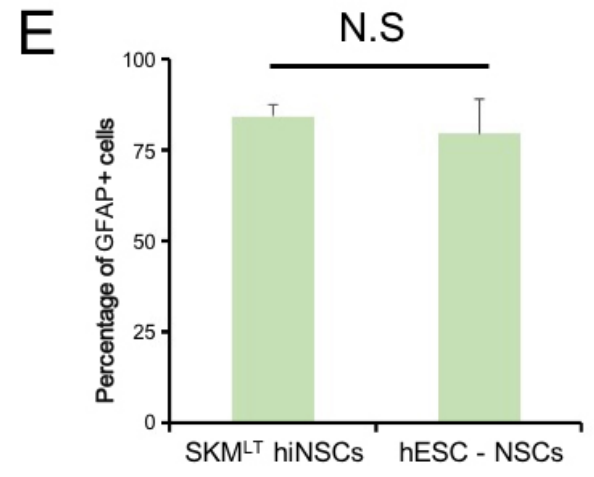

Fig. 4. Continued.

phology of NSCs with activation of NSC marker genes (Supplementary Fig. S3A and S3B). Among them, SKM ${ }^{\mathrm{LT}}$ hiNSC line 5 showed the strongest expression of NSC-related genes (Supplementary Fig. S3B) and thus, we selected this line for the rest of analysis. Similar with hESCderived NSCs, SKM ${ }^{\mathrm{LT}}$ hiNSCs exhibited the expression of NSC markers such as BRN2, BLBP, MSII, and MSI2 as evidenced by immunocytochemistry (Fig. 4A) and they also displayed the global gene expression pattern similar with that of hESC-derived NSCs as shown by RNA sequencing analysis (Fig. 4B). We also evaluate the in vitro differentiation potential of $\mathrm{SKM}^{\mathrm{LT}}$ hiNSCs by inducing differentiation into both neurons and glial cells. After two weeks of differentiation, SKM ${ }^{\mathrm{LT}}$ hiNSCs could differentiate into both $\mathrm{GFAP}^{+}$astrocytes and $\mathrm{TUF1}^{+}$neurons (Fig. 4C and 4D). SKM ${ }^{\mathrm{LT}}$ hiNSCs also exhibited a rare differentiation into oligodendrocytes (Supplementary Fig. S4A). Notably, the differentiation efficiency into each lineage was quite similar with those of hESC-derived NSCs (Fig. 4E and $4 \mathrm{~F}$ ), indicating that $\mathrm{SKM}^{\mathrm{LT}}$ hiNSCs are functionally comparable to hESC-derived NSCs. Finally, we also evaluated the in vitro differentiation potentials of hiNSCs into other neuronal subtypes. As a result, we found that SKM ${ }^{\mathrm{LT}}$ hiNSCs could differentiate into all major neuronal subtypes, namely GABAergic, glutamatergic, cholinergic, and dopaminergic neurons upon the prolonged culture of neurons derived from SKM ${ }^{\mathrm{LT}}$ hiNSCs as evidenced by immunostaining using antibodies against GABA, Glutamate, ChAT (choline acetyltransferase), and TH (tyrosine hydroxylase), respectively (Supplementary Fig. S4B). Altogether, our data indicate that $\mathrm{SKM}^{\mathrm{LT}}$ hiNSCs share key molecular and functional features with hESC-derived NSCs and they are indeed multipotent.

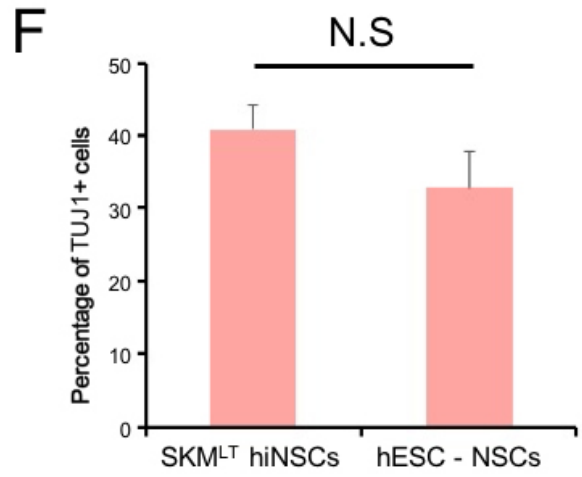

\section{Discussion}

Recent studies have described the generation of hiNSCs or induced neural progenitor cells by introducing defined sets of reprogramming factors $(18,21)$. Ring et al. (18) transduced single transcription factor, $S O X 2$, into human fetal fibroblasts in the presence of FGF2 and EGF to generate hiNSCs. Yu et al. (21) showed that the transduction of an aging-related factor, $H M G A 2$, together with SOX2 could enhance the hiNSC generation even from the senescent cells. On the other hand, it was also shown that the complete or partial sets of Yamanaka's factors that are generally used for iPSC generation could be also employed for generating NSC-like cell $(15,19,36)$. However, several technical limitations remain to be solved before translating hiNSC technology into the clinic. First, the direct conversion process toward hiNSCs using previous protocols is an extremely inefficient (0.003 to $0.96 \%)$ and time-consuming process (up to 35 days) (Supplementary Table S1) (15, 18, 19, 21, 22, 36). Second, Yamanaka factor-mediated iNSC generation step may involve a transient activation of pluripotency network, suggesting the potential tumorigenic nature of Yamanaka factor-mediated hiNSCs (17).

In contrast, $\mathrm{BSKM}^{\mathrm{LT}}$ could efficiently and reproducibly generate hiNSCs from multiple human somatic cell populations with distinct origins. $\mathrm{BSKM}^{\mathrm{LT}}$ combination showed the much higher reprogramming efficiency compared with previously described factor combinations. Throughout further optimization step, we could generate hiNSCs with either $\mathrm{SM}^{\mathrm{LT}}$ or $\mathrm{SKM}^{\mathrm{LT}}$, indicating that both BRN4 and KLF4 are not essential for hiNSC generation. Indeed, $\mathrm{SKM}^{\mathrm{LT}}$ combination exhibited the highest reprogramming efficiency (up to $2.4 \%$, around 80 times higher than OKSM) with accelerated conversion process (within 7 days) (Fig. 2B and 2D). This finding could be 
also explained by the key role of BRN4 in neuronal differentiation. BRN4 induces the differentiation of NSCs into neurons in an insulin-like growth factor-1 (IGF-1)dependent manner (37). This neurogenic role of BRN4 might decrease the yield of the self-replicative hiNSC population during $\mathrm{BSKM}^{\mathrm{LT}}$ mediated direct conversion process, also indicating the distinct role of BRN4 in induction of multipotency between human and mouse $(10,11,25)$.

Recently, we have described that BSKM-mediated iNSC production in mouse is a direct process which does not involve a transient acquisition of pluripotency (25). Thus, in contrast to Yamanaka factor-mediated direct conversion process, our direct conversion strategy is much more reliable and robust in the production of transplantable hiNSCs. However, there are still some hurdles remained for further translating our technology into the clinic. First, the cellular identity of transdifferentiated hiNSCs remains elusive. The unclear regional identity of hiNSCs might be caused by the heterogeneity of the reprogrammed cells (38) and thus, further clonal assay (23) for clearly addressing the regional identity of hiNSCs is highly required to elaborate cellular identity of hiNSCs for future application. Second, we observed that SV4OLT is essential for the efficient direct conversion toward hiNSCs, resulting in robust and reproducible hiNSC production. However, SV4OLT is a well-known oncogene capable of inducing malignant transformation of various cells. Thus, the residual expression of SV4OLT or integrated viral cassettes encoding SV4OLT should be fully eliminated in hiNSCs for therapeutic applications. Further efforts for inducing transient expression of SV4OLT without transgene integration such as an episomal vector system $(7,23)$ and in vitro transcribed $\mathrm{mRNA}$ are required. Third, insertional mutagenesis caused by the viral vector may lead to the continuous expression of exogenous reprogramming factors and the random gene-breaking in reprogrammed iNSCs, potentially resulting in functional defects (39). Therefore, as mentioned above, a safer method should be developed for integration-free gene delivery of exogenous reprograming factors.

Our study describes a novel protocol for robustly and reproducibly generating hiNSCs using a well-defined minimized gene set under specific culture conditions. Thus, this current protocol might be highly suitable for producing patient-specific hiNSCs for cell replacement therapy. Moreover, hiNSCs technology might be also useful for the precise in vitro modeling of various neuronal disorders and efficient screening of drug candidates.

\section{Acknowledgments}

This work was supported by National Research Foundation of Korea (NRF) grants funded by the Korean government (NRF-2016K1A3A1A61006005, NRF-2017M3C7A1047640) and also by a grant from the NextGeneration BioGreen 21 Program (PJ01322101), Rural Development Administration, Republic of Korea.

\section{Potential Conflict of Interest}

The authors have no conflicting financial interest.

\section{Supplementary Materials}

Supplementary data including two tables and four figures can be found with this article online at http://pdf. medrang.co.kr/paper/pdf/IJSC/IJSC-13-s19097.pdf.

\section{References}

1. Gage FH. Mammalian neural stem cells. Science 2000;287: 1433-1438

2. Blurton-Jones $M$, Kitazawa M, Martinez-Coria H, Castello NA, Müller FJ, Loring JF, Yamasaki TR, Poon WW, Green $\mathrm{KN}$, LaFerla FM. Neural stem cells improve cognition via BDNF in a transgenic model of Alzheimer disease. Proc Natl Acad Sci U S A 2009;106:13594-13599

3. Martino G, Pluchino $S$. The therapeutic potential of neural stem cells. Nat Rev Neurosci 2006;7:395-406

4. Xuan AG, Luo M, Ji WD, Long DH. Effects of engrafted neural stem cells in Alzheimer's disease rats. Neurosci Lett 2009;450:167-171

5. Takahashi K, Tanabe K, Ohnuki $M$, Narita $M$, Ichisaka T, Tomoda K, Yamanaka S. Induction of pluripotent stem cells from adult human fibroblasts by defined factors. Cell 2007;131:861-872

6. Lim KT, Kim J, Hwang SI, Zhang L, Han H, Bae D, Kim KP, Hu YP, Schöler HR, Lee I, Hui L, Han DW. Direct conversion of mouse fibroblasts into cholangiocyte progenitor cells. Stem Cell Reports 2018;10:1522-1536

7. Kim JH, Kim KP, Lim KT, Lee SC, Yoon JY, Song GQ, Hwang SI, Schöler HR, Cantz T, Han DW. Generation of integration-free induced hepatocyte-like cells from mouse fibroblasts. Sci Rep 2015;5:15706

8. Kim JB, Lee H, Araúzo-Bravo MJ, Hwang K, Nam D, Park MR, Zaehres H, Park KI, Lee SJ. Oct4-induced oligodendrocyte progenitor cells enhance functional recovery in spinal cord injury model. EMBO J 2015;34:2971-2983

9. Han DW, Greber B, Wu G, Tapia N, Araúzo-Bravo MJ, Ko K, Bernemann C, Stehling M, Schöler HR. Direct reprogramming of fibroblasts into epiblast stem cells. Nat Cell Biol 2011;13:66-71

10. Han DW, Tapia N, Hermann A, Hemmer K, Höing S, Araúzo-Bravo MJ, Zaehres H, Wu G, Frank S, Moritz S, Greber B, Yang JH, Lee HT, Schwamborn JC, Storch A, 
Schöler HR. Direct reprogramming of fibroblasts into neural stem cells by defined factors. Cell Stem Cell 2012;10: 465-472

11. Kim SM, Fla $\beta$ kamp H, Hermann A, Araúzo-Bravo MJ, Lee SC, Lee SH, Seo EH, Lee SH, Storch A, Lee HT, Schöler HR, Tapia N, Han DW. Direct conversion of mouse fibroblasts into induced neural stem cells. Nat Protoc 2014;9:871-881

12. Lim KT, Lee SC, Gao Y, Kim KP, Song G, An SY, Adachi K, Jang YJ, Kim J, Oh KJ, Kwak TH, Hwang SI, You JS, Ko K, Koo SH, Sharma AD, Kim JH, Hui L, Cantz T, Schöler HR, Han DW. Small molecules facilitate single factor-mediated hepatic reprogramming. Cell Rep 2016;15: 814-829

13. Hemmer K, Zhang $M$, van Wüllen T, Sakalem M, Tapia N, Baumuratov A, Kaltschmidt C, Kaltschmidt B, Schöler HR, Zhang W, Schwamborn JC. Induced neural stem cells achieve long-term survival and functional integration in the adult mouse brain. Stem Cell Reports 2014;3:423-431

14. Hong JY, Lee SH, Lee SC, Kim JW, Kim KP, Kim SM, Tapia N, Lim KT, Kim J, Ahn HS, Ko K, Shin CY, Lee HT, Schöler HR, Hyun JK, Han DW. Therapeutic potential of induced neural stem cells for spinal cord injury. J Biol Chem 2014;289:32512-32525

15. Lu J, Liu H, Huang CT, Chen H, Du Z, Liu Y, Sherafat MA, Zhang SC. Generation of integration-free and regionspecific neural progenitors from primate fibroblasts. Cell Rep 2013;3:1580-1591

16. Maza I, Caspi I, Zviran A, Chomsky E, Rais Y, Viukov S, Geula S, Buenrostro JD, Weinberger L, Krupalnik V, Hanna S, Zerbib M, Dutton JR, Greenleaf WJ, Massarwa R, Novershtern N, Hanna JH. Transient acquisition of pluripotency during somatic cell transdifferentiation with iPSC reprogramming factors. Nat Biotechnol 2015;33:769774

17. Bar-Nur O, Verheul C, Sommer AG, Brumbaugh J, Schwarz BA, Lipchina I, Huebner AJ, Mostoslavsky G, Hochedlinger $\mathrm{K}$. Lineage conversion induced by pluripotency factors involves transient passage through an iPSC stage. Nat Biotechnol 2015;33:761-768

18. Ring KL, Tong LM, Balestra ME, Javier R, AndrewsZwilling Y, Li G, Walker D, Zhang WR, Kreitzer AC, Huang Y. Direct reprogramming of mouse and human fibroblasts into multipotent neural stem cells with a single factor. Cell Stem Cell 2012;11:100-109

19. Zhu S, Ambasudhan R, Sun W, Kim HJ, Talantova M, Wang X, Zhang M, Zhang Y, Laurent T, Parker J, Kim HS, Zaremba JD, Saleem S, Sanz-Blasco S, Masliah E, McKercher SR, Cho YS, Lipton SA, Kim J, Ding S. Small molecules enable OCT4-mediated direct reprogramming into expandable human neural stem cells. Cell Res 2014;24: 126-129

20. Corti S, Nizzardo M, Simone C, Falcone M, Donadoni C, Salani S, Rizzo F, Nardini M, Riboldi G, Magri F, Zanetta C, Faravelli I, Bresolin N, Comi GP. Direct reprogramming of human astrocytes into neural stem cells and neurons.
Exp Cell Res 2012;318:1528-1541

21. Yu KR, Shin JH, Kim JJ, Koog MG, Lee JY, Choi SW, Kim HS, Seo Y, Lee S, Shin TH, Jee MK, Kim DW, Jung SJ, Shin S, Han DW, Kang KS. Rapid and efficient direct conversion of human adult somatic cells into neural stem cells by HMGA2/let-7b. Cell Rep 2015;10:441-452

22. Wang L, Wang L, Huang W, Su H, Xue Y, Su Z, Liao B, Wang H, Bao X, Qin D, He J, Wu W, So KF, Pan G, Pei D. Generation of integration-free neural progenitor cells from cells in human urine. Nat Methods 2013;10:84-89

23. Kim SM, Lim KT, Kwak TH, Lee SC, Im JH, Hali S, In Hwang S, Kim D, Hwang J, Kim KP, Chung HJ, Kim JB, Ko K, Chung HM, Lee HT, Schöler HR, Han DW. Induced neural stem cells from distinct genetic backgrounds exhibit different reprogramming status. Stem Cell Res 2016;16:460468

24. Yamashita T, Liu W, Matsumura Y, Miyagi R, Zhai Y, Kusaki M, Hishikawa N, Ohta Y, Kim SM, Kwak TH, Han DW, Abe K. Novel therapeutic transplantation of induced neural stem cells for stroke. Cell Transplant 2017;26:461467

25. Velychko S, Kang K, Kim SM, Kwak TH, Kim KP, Park C, Hong K, Chung C, Hyun JK, MacCarthy CM, Wu G, Schöler HR, Han DW. Fusion of reprogramming factors alters the trajectory of somatic lineage conversion. Cell Rep 2019;27:30-39.e4

26. Petersen GF, Strappe PM. Generation of diverse neural cell types through direct conversion. World J Stem Cells 2016;8:32-46

27. Li W, Sun W, Zhang Y, Wei W, Ambasudhan R, Xia P, Talantova M, Lin T, Kim J, Wang X, Kim WR, Lipton SA, Zhang K, Ding S. Rapid induction and long-term self-renewal of primitive neural precursors from human embryonic stem cells by small molecule inhibitors. Proc Natl Acad Sci U S A 2011;108:8299-8304

28. Xu Y, Wei X, Wang M, Zhang R, Fu Y, Xing M, Hua Q, Xie $\mathrm{X}$. Proliferation rate of somatic cells affects reprogramming efficiency. J Biol Chem 2013;288:9767-9778

29. Ruiz S, Panopoulos AD, Herrerías A, Bissig KD, Lutz M, Berggren WT, Verma IM, Izpisua Belmonte JC. A high proliferation rate is required for cell reprogramming and maintenance of human embryonic stem cell identity. Curr Biol 2011;21:45-52

30. Mirakhori F, Zeynali B, Rassouli H, Shahbazi E, Hashemizadeh S, Kiani S, Salekdeh GH, Baharvand H. Induction of neural progenitor-like cells from human fibroblasts via a genetic material-free approach. PLoS One 2015; 10:e 0135479

31. Zheng J, Choi KA, Kang PJ, Hyeon S, Kwon S, Moon JH, Hwang I, Kim YI, Kim YS, Yoon BS, Park G, Lee J, Hong $\mathrm{S}$, You S. Corrigendum to "A combination of small molecules directly reprograms mouse fibroblasts into neural stem cells" [Biochem. Biophys. Res. Commun. 476 (2016) 42-48]. Biochem Biophys Res Commun 2017;485:705

32. Park IH, Zhao R, West JA, Yabuuchi A, Huo H, Ince TA, Lerou PH, Lensch MW, Daley GQ. Reprogramming of hu- 
man somatic cells to pluripotency with defined factors. Nature 2008;451:141-146

33. Sgodda M, Mobus S, Hoepfner J, Sharma AD, Schambach A, Greber B, Ott M, Cantz T. Improved hepatic differentiation strategies for human induced pluripotent stem cells. Curr Mol Med 2013;13:842-855

34. Hoch RV, Lindtner S, Price JD, Rubenstein JL. OTX2 transcription factor controls regional patterning within the medial ganglionic eminence and regional identity of the septum. Cell Rep 2015;12:482-494

35. Gaber ZB, Butler SJ, Novitch BG. PLZF regulates fibroblast growth factor responsiveness and maintenance of neural progenitors. PLoS Biol 2013;11:e1001676

36. Mitchell RR, Szabo E, Benoit YD, Case DT, Mechael R, Alamilla J, Lee JH, Fiebig-Comyn A, Gillespie DC, Bhatia M. Activation of neural cell fate programs toward direct conversion of adult human fibroblasts into tri-potent neural progenitors using OCT-4. Stem Cells Dev 2014;23:19371946

37. Shimazaki T, Arsenijevic Y, Ryan AK, Rosenfeld MG, Weiss S. A role for the POU-III transcription factor Brn-4 in the regulation of striatal neuron precursor differentiation. EMBO J 1999;18:444-456

38. Francesconi M, Di Stefano B, Berenguer C, de AndrésAguayo L, Plana-Carmona M, Mendez-Lago M, Guillaumet-Adkins A, Rodriguez-Esteban G, Gut M, Gut IG, Heyn H, Lehner B, Graf T. Single cell RNA-seq identifies the origins of heterogeneity in efficient cell transdifferentiation and reprogramming. Elife 2019;8:e41627

39. Kang X, Yu Q, Huang Y, Song B, Chen Y, Gao X, He W, Sun X, Fan Y. Effects of integrating and non-integrating reprogramming methods on copy number variation and genomic stability of human induced pluripotent stem cells. PLoS One 2015;10:e131128 